\title{
Photodynamics: How Massive Photons, Gravitons, Gluons, and Neutrinos Manage to Travel at the Speed of Light
}

\section{Robert J Martineau*}

NASA Goddard Space Flight Centre, Greenbelt, MD 20771-0003, USA

\begin{abstract}
In this paper we introduce Photodynamics and use it to resolve three outstanding problems in physics: in relativity, the motion of massive particles at the speed of light; in cosmology, how cooling CMB photons lose energy for 13.8 billion years without slowing down; in neutrino astrophysics, neutrino oscillation at light speed in response to the challenge posed by the 2015 Nobel Prize in Physics. These are all major unsolved problems in relativity, cosmology, and neutrino astrophysics, whose solutions have evaded our best efforts for decades. The common cause of these problems has been the erroneous application of Einstein's $E=m c^{2}$ to particles that travel at the speed of light. We prove conclusively that $E=m c^{2}$ applies only to particles that cannot travel at the speed of light, and in fact prevents them from attaining light speed. This reveals that an enormous problem has existed, over a century. Relativity, the foundation of modern physics, is woefully incomplete and inadequate. It does not contain a valid relativistic dynamics for the vast majority of particles in the Universe, the particles that travel at the speed of light. To resolve this problem we introduce Photodynamics, a previously unknown fundamental law of nature. Photodynamics is the only mechanism that allows particles to travel at the speed of light, and then, only if they have mass. This means that photons, gravitons, gluons, and neutrinos are all relativistic massive particles. So all theories describing the origin, characteristics, and interactions of fundamental particles as well as those of the origin, constitution, and evolution of the Universe, must be made compatible with photodynamics. Photodynamics is a change in relativity, a change in the very foundations of physics, and as such will impact all fields of physics involving particles that travel at the speed of light.
\end{abstract}

Keywords: Relativity; Self-propulsion; E $=\mathrm{mc}^{2}$; Neutrino oscillation; Light speed; $\mathrm{CMB}$ photon cooling

\section{Introduction}

As the best known particle in its class, we use photons to develop Photodynamics, but it applies to all particles that travel at the speed of light. The photon is the most abundant and readily available elementary particle we know, and yet it is arguably still the most mysterious and ill understood. We do know that it has a frequency $\omega_{0}$, an energy $\hbar \omega_{0}$, and an angular momentum $\hbar$ either aligned or anti-aligned with its direction of motion. On the other hand, there are a number of troubling, unanswered questions, and inconsistencies with our present understanding of the photon. Let us list a few of these. Most particles can travel at different speeds, but the photon cannot. Why? How can a photon go from zero to the speed of light when a flashlight is turned on without going at any speed in between? Using $\mathrm{E}=m \mathrm{c}^{2}$ a photon is seen to have a constant mass $m=\hbar \omega_{0} / c^{2}$ and travels at the speed of light. Why can't atomic particles with the same constant mass go at the speed of light? The energy of most particles depends on their speeds, but the photon's energy does not. Why? A photon can lose energy for 13.8 billion years and keep going at the speed of light (CMBR) [1-3]. Why? What causes the photon to travel at the speed of light, and what is the physical mechanism which prevents the photon from going faster than the speed of light? The photon's energy is $\hbar \omega_{0}$. What is revolving at angular frequency $\omega_{0}$ ? What determines the direction of flight? Why is the photon's angular momentum either aligned or anti-aligned with its flight axis? If something is indeed revolving, how far is it from the axis of rotation? What is its orbital speed? Why is it revolving at that distance from the axis, and at that speed, rather than at a different distance and speed?

In the area of neutrino astrophysics, neutrinos have been shown to undergo flavour oscillation while traveling at the speed of light. Neutrino oscillation cannot happen unless neutrinos have a non-zero rest mass as recently highlighted by the 2015 Nobel Prize in physics. How is this possible using $\mathrm{E}=\mathrm{mc}^{2}$ ?
The plan of the paper is as follows: We begin with neutrino oscillation and its conflict with relativity, in particular $\mathrm{E}=\mathrm{mc}^{2}$. This leads us to examine the validity of using $\mathrm{E}=\mathrm{mc}^{2}$ for particles, like the neutrino, that travel at the speed of light. We prove that $\mathrm{E}=\mathrm{mc}^{2}$ and its associated energy-momentum theorem do not apply to any particle that travels at the speed of light, and that, consequently, relativity is incomplete. As presently formulated, it does not possess valid dynamics for particles that travel at the speed of light. This is an unexpected shortcoming of enormous importance.

The Universe is composed of only two types of particles, those that can and those that cannot travel at the speed of light, and we don't know the laws that govern the motion of half these particles by type, and the vast majority numerically! To solve this problem we derive the laws of Photodynamics. These laws not only provide the missing dynamics, but also reveal that particles like the photon possess exciting new properties, one of which is self-propulsion. We use self-propulsion to explain how $\mathrm{CMB}$ photons lose energy for 13.8 billion years without slowing down. We then examine the origin of the photon's angular momentum and angular frequency, summarize our findings, and offer concluding remarks.

In somewhat more detail, we begin by giving a short history of neutrino astrophysics to show what gave rise to the neutrino oscillation

*Corresponding author: Robert J. Martineau, NASA Goddard Space Flight Center, Greenbelt, Md. (retired 2006), 20771-0003, USA, Tel: 1-352-746-2396; E-mail: rjmhere@yahoo.com

Received November 21, 2018; Accepted December 20, 2018; Published December 27, 2018

Citation: Martineau RJ (2018) Photodynamics: How Massive Photons, Gravitons, Gluons, and Neutrinos Manage to Travel at the Speed of Light. J Phys Math 9: 293 doi: 10.4172/2090-0902.1000293

Copyright: @ 2018 Martineau RJ. This is an open-access article distributed under the terms of the Creative Commons Attribution License, which permits unrestricted use, distribution, and reproduction in any medium, provided the original author and source are credited. 
hypothesis. We describe the success of the theory and the problem associated with the phenomenon. The problem is a conflict between the theory and experimental facts of neutrino oscillation, and relativity as presently formulated, in particular $\mathrm{E}=\mathrm{mc}^{2}$. Application of $\mathrm{E}=\mathrm{mc}^{2}$ to massive lightspeed neutrinos leads to all neutrinos having infinite energy. To resolve this problem, we demonstrate that $\mathrm{E}=\mathrm{mc}^{2}$ does not apply to any particle that travels at the speed of light. Since neutrinos travel at the speed of light, this resolves the neutrino oscillation problem. However, this solution reveals the existence of a far greater problem, one which has existed, unknown, for over a century. Relativity, the foundation of modern physics, is woefully incomplete and inadequate. It does not contain a valid relativistic dynamics for particles that travel at the speed of light.

The resolution of this problem requires a fundamental change in relativity, one which in fact comprises and justifies our introduction of the laws of Photodynamics. These laws are the relativistically invariant dynamical equations which explain how certain massive particles manage to travel at the speed of light. Photodynamics also reveals that these particles possess new properties and extraordinary capabilities never before imagined.

One of these capabilities is that of self-propulsion. The new energy-momentum theorem associated with Photodynamics has the extraordinary property that if a particle were to slow down, its energy would increase. We demonstrate that, akin to rocket propulsion in space to its terminal velocity, a photon consumes its internal energy to generate forces that accelerate it in the direction of flight until all its internal energy has been consumed and it has reached its terminal velocity, the speed of light. We use this newly discovered property of photons to explain how CMB photons can lose energy for 13.8 billion years without slowing down.

Next we examine the photon in its rest frame, that is, the frame in which it has no translational motion. We show why the direction of its translational motion, plus or minus, is determined by its angular momentum. We address the nature of the photon's angular momentum and the origin of its frequency, $\omega_{0}$, without which it would not exist.

Finally we end with a list and ranking of significant findings, and provide concluding remarks on the expected impact of these developments.

\section{Neutrino Oscillation versus Relativity}

Neutrinos and antineutrinos come in three flavours: electron, muon, and tau. In the 1960's there arose a problem with the measured electron neutrino flux from the sun known as the solar neutrino problem [4]. This problem refers to a deficiency in the measured solar neutrino flux first discovered in the Homestake Experiment [5-7]. The measured flux was about $1 / 3$ the expected value and was later confirmed to be $1 / 3$ by the Kamioka and Sudbury Observatories. The solution of this problem came about after Pontecorvo [8-10] suggested, in 1967, that neutrinos could change flavour in mid flight if they possessed nonzero rest masses. Since only electron flavoured neutrinos were being measured, this explained the $1 / 3$ factor observed. This phenomenon, called neutrino oscillation [11], provided a solution to the solar neutrino problem [12]. Neutrino oscillation was subsequently experimentally confirmed in 1998 and 2001 [13], and was acknowledged as a great discovery in neutrino astrophysics by the award of the 2015 Nobel Prize in physics for the experimental confirmation of neutrino oscillation "which shows that neutrinos have mass". However, it led to an even greater problem by confirming that neutrinos, known to travel at the speed of light, have mass.
Even though neutrino oscillation was needed to resolve the solar neutrino problem, it requires neutrinos to have non-zero rest masses while traveling at the speed of light, something not allowed by Einstein's $\mathrm{E}=\mathrm{mc}^{2}$. This conflict between mass, motion, and relativity is called the neutrino oscillation problem.

For decades, based on repeated experimental measurements, neutrinos were said to travel at the speed of light. When one measures the speed of light, one always gets $c$ within experimental error, not something else. The same thing happens when one measures the speed of neutrinos. As these experiments got better and better, the experimental errors got smaller and smaller. Measurements from Fermilab [14] yielded a precision of $4 \times 10^{-5}$, and those from Supernova 1987A [15] a precision of $2 \times 10^{-9}$, that is 1.000000002 times the speed of light. In addition, one could ask the question, "Where have all the slow neutrinos gone?" There are no slow neutrinos, just like there are no slow photons, and for the same reason: both go at the speed of light. For these reasons, the speed of neutrinos, in the Standard Model, was for years stated to be the speed of light, and because of relativity, its rest mass was said to be zero. Now, hiding behind ever present experimental error, neutrinos are said to be massive, and to travel very, very close to the speed of light, but not at the speed of light. The experimental fact that neutrinos travel at the speed of light is being denied to preserve relativity. Avoidance of the conflict between neutrino oscillation and relativity by denying the experimental facts puts physics in a bad light and is unacceptable. This decades old conflict has become a glaring problem of current interest because of the recent Nobel Prize, and cries out for resolution.

We will resolve this longstanding problem by providing a rigorous mathematical proof that $\mathrm{E}=\mathrm{mc}^{2}$ does not apply to any particle that travels at the speed of light. Thus massive neutrinos can travel at the speed of light in conformity with the experimental facts, without violating relativity, if $\mathrm{E}=\mathrm{mc}^{2}$ is not misapplied.

We next describe the historical error which led to the use of $\mathrm{E}=\mathrm{mc}^{2}$ and its associated energy momentum theorem on particles that travel at the speed of light and then derive the correct dynamics for these particles.

\section{An Historical Error}

We know how massive objects acquire speed. For example, let's put a cartridge in a rifle and fire it. When the powder explodes it provides energy for the explosive force to accelerate the bullet to its terminal velocity. For this to happen we need an external source of energy, and an external force to increase the momentum and energy of the bullet. The rifled barrel confines the energy, except in one direction. The bullet will in general encounter external forces, and if fired up will lose energy and speed. A photon is not like that. After being emitted upward by an atom, it keeps going. It loses energy but maintains its speed for vast distances, even inter-galactic distances. We see this in the Cosmic Microwave Background Radiation when we observe the oldest light in the universe, what has been called the very first light [3]. These photons have been traveling for 13.8 billion years, and although their energy has been enormously degraded, they continue to travel at the speed of light. In order to replicate this feat, a bullet would have to carry with it an imaginary gun barrel, and charges it could fire to maintain speed every time it slows down.

So why is a photon so different? Is it because it has zero rest mass? Does having a zero rest mass constitute a precondition for attaining 
and maintaining the speed of light? The answer to this question is an emphatic no. In fact, the opposite is the case.

In order to understand how the physics community came to the conclusion that the photon has a zero rest mass and can only travel at the speed of light, we must examine the Einstein relation in more detail. Recall that the Einstein energy-momentum theorem is given by:

$$
E_{u}^{2}-c^{2} \bar{P}_{u}^{2}=m_{0}^{2} c^{4}
$$

where

$$
E_{u}=m c^{2}=m_{0} c^{2} \gamma, \bar{P}_{u}=m_{0} \bar{u} \gamma
$$

and $\gamma$ is the Lorentz factor $\gamma^{-1}=\sqrt{1-u^{2} / c^{2}}$. If we take the time derivative of eqn. (1), we get:

$$
\frac{d E_{u}}{d t}=\bar{u} \cdot \bar{F}_{u}
$$

where the force $\bar{F}_{u}$ is as usual:

$$
\bar{F}_{u}=\frac{d \bar{P}_{u}}{d t}
$$

If the applied force $\bar{F}_{u}$ is in the direction of $\bar{u}$, it does positive work on the particle increasing both its momentum and energy to maintain the invariant energy of the energy-momentum theorem. These processes are understood and verified for all particles that can't travel at the speed of light. The energy momentum theorem is the keystone of particle dynamics, telling us how these particles behave in the classical and relativistic domains. This is why the Einstein relation, $\mathrm{E}=\mathrm{mc}^{2}$, has a deservedly high reputation and was applied to the photon in an attempt to determine its properties. Besides, there was no available alternative.

The historical argument proceeds as follows. Either the rest mass of the photon is zero or non-zero. If the rest mass of the photon were non-zero, then $E=m c^{2}=m_{0} c^{2} \gamma$ would be infinite at light speed, and all photons would have infinite energy since $\gamma$ is infinite at light speed. Therefore, it was concluded, the rest mass of the photon must be zero. Furthermore, if $\mathrm{m}_{0}$ is zero, then $E$ is clearly zero for all speeds with the possible exception of $\mathrm{u}=\mathrm{c}$ where $E$ supposedly becomes indeterminate due to the fact that $\lim _{u \rightarrow c} m_{0} \gamma \rightarrow 0 \times \infty$. The thinking is that since $E$ is indeterminate at lightspeed, it could conceivably be the energy of a photon. This reasoning led to the present consensus belief that the photon has a zero rest mass, and can only travel at the speed of light. This is also what was expected for the neutrino, before neutrino oscillation, which cannot occur if $\mathrm{m}_{0}=0$.

At first sight this seems to be true, since putting $\mathrm{m}_{0}=0$ into eqn. (1) gives $E=c P$, and therefore:

$$
E=\hbar \omega=c P .
$$

This together with $P=\hbar k$ yields the well known relation between a photon's speed, wavelength, and frequency $c=\omega / k=\lambda \nu$. In addition, for a photon, since energy has a mass equivalence, we could write, and often see:

$$
E=\hbar \omega=m c^{2}=c P
$$

where $m$ is a constant, which we are told cannot be called $m_{0}$ because, supposedly, we can't get $\mathrm{E}=\mathrm{cP}$ without saying $\mathrm{m}_{0}=0$. This reasoning is wrong. As we will see, the correct form of eqn. (6) is:

$$
E=\hbar \omega_{0}=m_{0} c^{2}=c P_{0},
$$

where $m_{0} \neq 0$. And using Einstein's $E=m c^{2}$ yields eqn. (5) only if
$E=\hbar \omega=c P=0$ as we will shortly see.

Still, the consensus conclusion, based on the reasoning presented above, is that the photon has zero rest mass and can only travel at the speed of light. However appealing and justified these findings appear to be, and they are held by the majority of the physics community, they are wrong, as we will now demonstrate.

Application of $\mathrm{E}=\mathrm{mc}^{2}=\mathrm{m}_{0} \mathrm{c}^{2} \gamma$ to the photon definitely requires $\mathrm{m}_{0}$ to be zero to avoid infinite energies at light speed. Then if $\mathrm{m}_{0}=0$ and $\mathrm{u} \neq \mathrm{c}, \mathrm{E}$ is also zero, so these speeds are not allowed. That only leaves $\mathrm{u}=\mathrm{c}$. This is the traditional reason for the conclusion that the photon has a zero rest mass and can only travel at the speed of light. But let us take a closer look at $E$ as u approaches $c$. We have seen that this limit results in the indeterminate form:

$$
\lim _{u \rightarrow c} \cdot\left(m_{0} \gamma\right)=\lim _{u \rightarrow c} \cdot(0 \times \gamma)=\lim _{u \rightarrow c} \cdot\left(\frac{0}{\gamma^{-1}}\right)=\frac{0}{0} .
$$

But $0 / 0$ is indeterminate only if we don't know the numerator or the denominator. If we do, then forms like $x / x, x^{2} / x, x / x^{2}$ and $\sin (x) / x$ are not indeterminate at $\mathrm{x}=0$ but go respectively to $1,0, \infty, 1$. In general, to resolve "indeterminate" forms like these we use l'Hospital's rule and take the ratio of the derivatives of the numerator and denominator, both evaluated at the limit. If we do this in eqn. (8), we get:

$$
\lim _{u \rightarrow c} \cdot\left(\frac{0}{\gamma^{-1}}\right)=\lim _{u \rightarrow c} \cdot\left(\frac{0^{\prime}}{\left(\gamma^{-1}\right)^{\prime}}\right)=\lim _{u \rightarrow c} \cdot\left(\frac{0}{-u \gamma / c^{2}}\right)=-\left(\frac{0}{\infty}\right)=0
$$

where (') means a derivative with respect to $\mathrm{u}$. Therefore $E$ is always zero, and the photon does not exist if $\mathrm{m}=0$, for any $\mathrm{u}$, even $\mathrm{u}=\mathrm{c}$. We conclude, using this relation, that particles that travel at the speed of light cannot exist, even if $\mathrm{m}_{0}=0$. According to this argument, if we use $\mathrm{E}=\mathrm{mc}^{2}$, a particle that travels at the speed of light is not allowed to have a zero rest mass, nor is it allowed to have a non-zero rest mass, so it cannot exist. In addition, because $E=\hbar \omega_{0}$, the photon has a mass $m_{0}=\hbar \omega_{0} / c^{2}$ travelling at the speed of light, something not allowed by Einstein's energy relation. This can only mean that $\mathrm{E}=\mathrm{mc}^{2}$ has limited validity. $\mathrm{E}=\mathrm{mc}^{2}$ applies to particles that can't travel at the speed of light, but not to those that can.

Our proof extends to the invariant Einstein energy-momentum theorem, eqn. (1). Since neither $E$ nor $\bar{P}$ can be used to describe particles that travel at the speed of light (both have $m_{0} \gamma$ factors), neither can the Einstein energy-momentum theorem itself. Nevertheless, it has often been used to "prove" that photons are massless. By putting $\mathrm{m}_{0}=0$ into eqn. (1), one gets $\mathrm{E}=\mathrm{cP}$, a well known expression in traditional photonics, thus seemingly confirming the fact that photons have a zero rest mass. However, putting $\mathrm{m}_{0}=0$ into eqn. (1) yields $\mathrm{E}=\mathrm{cP}$ only because both $\mathrm{E}$ and $\mathrm{P}$ are zero since each contains an $\mathrm{m}_{0} \gamma$ factor which is zero at light speed if $\mathrm{m}_{0}=0$.

To summarize, the fundamental error that has been made has been the application of $\mathrm{E}=\mathrm{mc}^{2}$ to the photon. This has been a mistake of historic proportion, lasting for over a century to the present day, and can no longer be tolerated. The veneration given to this famous equation, then and now, drove, sustained, and continues to motivate the belief that photons have zero rest mass and can only travel at the speed of light.

We can only conclude that since it was first formulated, relativity has never contained a valid relativistic dynamics for particles that travel at the speed of light. This is very important since the Universe is composed of only two types of particles: those that can travel at the 
speed of light and those that can't. Of these, light speed particles are in the vast majority. For example, for each atomic particle there are about 10 billion photons, not to mention other light speed particles. Unfortunately, the role of light speed particles is widely overlooked and unappreciated. One should never forget that the photon, gluon, and graviton mediate the electromagnetic, strong, and gravitational forces. The neutrino plays an important role in the workings of the weak force. These fundamental forces make the existence of the Universe possible, and determine its contents, properties, structure, and evolution. So the proper dynamics of light speed particles must be determined and understood if the Universe is to be understood. For over 100 years the Einstein equations have been erroneously applied to particles like photons, gravitons, gluons, and neutrinos leading to the institutionalization of numerous errors and the generation of several intractable problems.

We now turn our attention to deriving the missing dynamics, which we call Photodynamic after the most visible particle of its type, the photon.

\section{Photodynamics}

We have concluded that $\mathrm{E}=\mathrm{mc}^{2}$, and the associated energymomentum theorem of eqn. (1), do not apply to the photon and other particles that travel at the speed of light. A new energy-momentum theorem is needed. Contrary to expectation, it is possible to derive a relativistic, invariant energy momentum theorem for particles that travel at the speed of light and also have a non-zero rest mass. In fact, the derivations of the two invariant energy-momentum theorems are closely related. They both originate from using the Lorentz factor $\gamma$ to derive quadratic invariants in $E$ and $P$. The fact that there are only two ways to do this, and that the Universe has only two types of particles is not a coincidence. We can write the Lorentz factor in the form:

$$
\gamma_{u}^{2}\left(1-\frac{\bar{u}^{2}}{c^{2}}\right)=1
$$

or

$$
\frac{1}{\gamma_{u}^{2}}+\frac{\bar{u}^{2}}{c^{2}}=1
$$

If we multiply eqn. (10) by $\mathrm{E}_{0}{ }^{2}=\mathrm{m}_{0}{ }^{2} \mathrm{c}^{4}$, we get the Einstein energymomentum theorem given in eqn. (1), and the usual definitions found in eqn. (2). If we multiply eqn. (11) by $\mathrm{e}_{0}{ }^{2}=\mathrm{m}_{0}{ }^{2} \mathrm{c}^{4}$, we get:

$$
e_{u}^{2}+c^{2} \bar{p}_{u}^{2}=e_{0}^{2},
$$

where

$$
e_{u}=\frac{e_{0}}{\gamma_{u}}, \bar{p}_{u}=m_{0} \bar{u} \text { and } \mathrm{e}_{0}=\mathrm{m}_{0} \mathrm{c}^{2} .
$$

Eqn. (12) is a new, relativistic, invariant energy-momentum theorem. It is a new fundamental law of nature that governs the motion of photons and other particles that travel at the speed of light. Notice that infinite energies and momenta do not occur at light speed, even though $\mathrm{m}_{0} \neq 0$. We call particles that have a non-zero rest mass and travel at the speed of light type 1 particles, and particles that obey Einstein's energy-momentum theorem type 2 particles. Photons, gravitons, gluons, and neutrinos are examples of type 1 particles. In the normal world, type 2 particles and bodies appear to vastly outnumber type 1 particles, but that is not the case. The number of photons in the Universe vastly outnumbers the number of atomic particles, not even counting the neutrinos, gravitons, and gluons. And if type 1 particles are more fundamental, and type 2 particles consist of type 1 particles at a lower level, then the ratio of type 1 to type 2 particles becomes even greater.

We have seen that there are two ways the Lorentz factor can be written to yield invariant kinematic identities and their associated energy-momentum theorems in the derivations of eqns. (1) and (12). This is why there are two types of particles, and two energy relations which we can write as $E_{1}=m_{1} c^{2}$, and $E_{2}=m_{2} c^{2}$ where, $m_{1}=m_{0} \gamma^{-1}$ and $m_{2}=m_{0} \gamma$. The new energy relation, $E_{1}=m_{1} c^{2}$, is as important as the Einstein relation, $\mathrm{E}_{2}=\mathrm{m}_{2} \mathrm{c}^{2}$, and is indispensable for the complete description of nature. If type 2 particles are composed of type 1 particles at a lower stage, it would turn out to be much more fundamental.

There is nothing preventing very massive particles from traveling at the speed of light. It is entirely possible that some seldom seen but well known massive particles actually travel at the speed of light although this is not even suspected because of their huge masses. Among these, the $\mathrm{W}$ and $\mathrm{Z}$ bosons could conceivably use Photodynamic to mediate the weak force like the photons, gluons, and gravitons use it to mediate the other fundamental forces.

The plus sign preceding the momentum in eqn. (12) as opposed to the minus sign in eqn. (1) provides the new energy-momentum theorem with unique, remarkable properties, as we will see.

In eqn. (12) we call $e_{u}$ the internal energy or relativistic energy of the photon. It is also useful to think of $e_{u}$ as the rest energy of the photon, or what remains of it if $\bar{u}$ is not zero. We sometimes refer to $\mathrm{cp}_{\mathrm{u}}$ as the photon's energy of motion. We notice that the internal energy of a photon takes on its maximum value $e_{u}=e_{0}$ when it is at rest. As its internal energy decreases, it gains speed, and its momentum increases until $\mathrm{e}_{\mathrm{u}}=0$ and $\mathrm{p}_{\mathrm{u}}=\mathrm{m}_{0} \mathrm{c}$. This is achieved without having to say $\mathrm{m}_{0}=0$.

The need to understand and assimilate this unusual behaviour cannot be overemphasized. Obviously eqn. (12) does not apply to normal massive particles whose relativistic energies and momenta both go to infinity as velocities approach the speed of light.

If one takes a time derivative of eqn. (12) when $\mathrm{m}_{0}$ is a constant, one obtains:

$$
\frac{d e_{u}}{d t}=-\gamma_{u} \bar{u} \cdot \bar{f}_{u} \text { where } \bar{f}_{u}=\frac{d \bar{p}_{u}}{d t}
$$

is the force acting on the particle.

One might be tempted to say that when $\bar{f}_{u}$ is in the direction of a photon's motion, it does positive work on the photon, increasing its speed and energy. That is not what happens. According to eqn. (14), positive $\bar{u} \cdot \overline{f_{u}}$ will increase the photon's speed, but will decrease its energy. If $\bar{f}_{u}$ were an opposing force, the photon's speed would be decreased and its energy increased. This unexpected result, caused by the difference in sign of the momenta in the energy-momentum theorems, is remarkable and leads to an entirely new particle dynamics. We call the new dynamics Photodynamics. It is the dynamics of type 1 particles, that is, of particles governed by eqns. (12), (13), and (14).

We notice that type 1 particles, like the photon, can travel at any speed from zero to the speed of light along a particular direction $\hat{u}$. If $\bar{u}=0$, the photon is at rest and has its maximum energy $\mathrm{e}_{0}=\mathrm{m}_{0} \mathrm{c}^{2}$. If a photon is travelling at the speed of light, then $\mathrm{e}_{\mathrm{u}}=0$, and $c P_{0}=E_{0}=\hbar \omega_{0}=m_{0} c^{2}$. This is eqn. (7). Since $\mathrm{E}=\mathrm{cP}$, we see that all existing photonics based on $E=\hbar \omega_{0}=c P$ is covered by photodynamics when $\mathrm{u}=\mathrm{c}$. Photodynamic is the sole, internally consistent theory of traditional photonics based on $\mathrm{E}=\mathrm{cP}$. It does not exist if $\mathrm{m}_{0}=0$. 
When an atom emits a photon, a quantum mechanical process is at work which also creates the photon's angular momentum at the same time. A photon can't exist without having an angular momentum $\pm \hbar$ aligned with its direction of flight. In fact, we will see that it is the photon's angular momentum that determines the flight axis. Upon emission, the photon experiences a force which accelerates it to the speed of light. According to eqn. (14) the force on the photon increases the photon's momentum and decreases its internal energy until $e_{u}=0$ and $\mathrm{p}_{\mathrm{u}}=\mathrm{m}_{0} \mathrm{c}$ along the direction of flight. In essence, the speed of the photon's rest mass goes from essentially zero, the atom's relative speed, to the speed of light in a very short period of time. The internal energy $\mathrm{e}_{\mathrm{u}}=\mathrm{m}_{0} \mathrm{c}^{2}$ becomes identified with photon energy $\hbar \omega_{0}$ per eqn. (7). But where does $\omega_{0}$ come from? What revolves or oscillates with angular frequency $\omega_{0}$ ? We are not talking about an electromagnetic field with a lot of waves. We are talking about a single photon, which is moving along a given axis at a steady speed, c. Before we answer this question, let us first present the most remarkable feature of Photodynamics, that of self-propulsion.

\section{Self-propulsion}

Self-propulsion is a remarkable new property associated with Photodynamics. We can write energy momentum theorems like eqn. (12) for each axis separately. For the z-axis this would be $e_{z}^{2}+c^{2} p_{z}^{2}=e_{0}^{2}$. If a photon is at rest, then $\mathrm{e}_{\mathrm{z}}=\mathrm{e}_{0}$, and $\mathrm{cp}_{\mathrm{z}}=0$. If the photon is traveling at the speed of light, then $\mathrm{e}_{\mathrm{z}}=0$, and $\mathrm{cp}_{\mathrm{z}}=\mathrm{e}_{0}$. Since a photon can travel at any speed between 0 and the speed of light, why does a photon usually travel at the speed of light? It does this because entropy subjects any non-zero internal energy to energy minimization causing it to quickly decay to zero and in the process to generate an internal force which accelerates the photon to the speed of light. This behaviour is given by eqn. (14). We call this behaviour self-propulsion because it occurs in the absence of any external force. So if a photon is emitted at a speed less than the speed of light, it will accelerate itself quickly to the speed of light.

We should point out that self-propulsion is a property of type 1 particles only. For type 2 particles, if $\bar{f}_{u}$ is an internal force, and $\bar{u} . \bar{f}_{u}>0$, the force will increase both the particle's energy, $E_{u}$, and its energy of motion, $c P_{u}$. Where does the energy come from if there is no external agent? Likewise, if the force is a negative force, both the energy, $E_{u}$, and the energy of motion, $c P_{u}$, are decreased. Where does this energy go? For this to happen, $\bar{f}_{u}$ must be an external force which can supply or sink the energy in play. Type 1 particles have built in sources and sinks of energy which allow the force to be an internal force.

From eqns. (12) and (14) we also note that the process of selfpropulsion is non-dissipative. The internal force, $\bar{f}_{u}$ can increase or decrease the photon's internal energy and energy of motion. Whatever increase in internal energy a photon experiences comes from a decrease in the photon's energy of motion and vice versa. None of the energy is lost. The process is like that of a vehicle with a battery, an electric engine, and reactive brakes which recharge the battery. The big difference is that the photon, as an engine, is simple, has no moving parts, and is non-dissipative. Even if the photon loses some mass, $\Delta \mathrm{m}_{0}$, due to some external force, short of hitting a brick wall, it always has enough $\mathrm{m}_{0}$ to reach the speed of light. In case of mass loss, eqn. (12) still holds, but with reduced mass, so self-propulsion continues to apply. No wonder a photon can travel vast distances, even inter-galactic distances, and keep going at the speed of light even though it loses some energy to external forces along the way. Self-propulsion is an unexpected and truly remarkable property of Photodynamics.
To get some idea of how quickly and forcefully self-propulsion works, we examine the motion of a photon along its flight axis, which we take as the $\mathrm{z}$-axis. It turns out, as we will see, that angular momentum prevents free flight along the $\mathrm{x}, \mathrm{y}$ axes or simultaneous selfpropulsion along any and every direction. This prevents the photon from blowing up. If a photon is emitted at $\mathrm{z}=0$ with zero velocity, a possible motion is given by $\dot{z}^{\prime}=c \tanh \omega_{0} t^{\prime}$. An estimate of how quickly the photon gains speed by self-propulsion is given by the time constant $1 / \omega_{0}$. For blue light, this corresponds to about $10^{-14}$ seconds. After a few time constants, the photon is traveling at the speed of light for all practical purposes. Likewise, an estimate of the acceleration of the photon is given by $c \omega_{0}$. This is orders of magnitude greater than normally encountered accelerations, like for example, the acceleration due to gravity. The corresponding internal force is orders of magnitude greater than that of the force of gravity. Self-propulsion is fundamental to the proper understanding of photon flight as well as the flight and interaction properties of all type 1 particles.

\section{The CMBR Photon Cooling Problem}

Cosmic Microwave Background photons have been participating in the expansion of the universe for 13.8 billion years, constantly losing energy without slowing down. In this process, their blackbody temperature has cooled from thousands of degrees to $2.74^{\circ} \mathrm{K}$ as measured by the COBE experiment. How photons can lose energy without slowing down is a mystery which has never been explained. The lack of a cogent physical explanation for this phenomenon is called the CMB photon cooling problem.

Some, as usual, say there is no problem. Photons continue to travel at the speed of light because they are photons. That is what they do. This of course is not a physical explanation of the phenomenon, but more of an incantation based on the invalid application of the Einstein equations. The phenomenon just cannot be explained by present day physics.

To get a better idea of the problem, let us take a physical example. Fire a bullet vertically into the sky. We find that in rising vertically it does work against the gravitational force and in the process must constantly supply energy to the gravitational field. To do this it uses its kinetic energy and slows down until all its kinetic energy has been used up and it momentarily comes to rest at the top of its trajectory. Now the force of gravity does positive work on the bullet as it falls and, neglecting friction, gives it back all the energy it lost on the way up. If photons obeyed the same laws of physics, and they are supposed to, they would do the same thing. But they don't. So we have a problem. We now know the solution to this problem. Photons obey different laws. They obey the laws of Photodynamics.

We know that self-propulsion acts quickly and forcefully to drive photons to achieve and maintain the speed of light. If a photon traveling at the speed of light has to supply energy to its environment in order to proceed, it must find another way to do this besides slowing down. Because of self propulsion, slowing down is not an option. Let us examine another option, the only one that remains. The momentum of a photon traveling in any direction at the speed of light is $p=m_{0} c$. Imagine that as it travels it is opposed by an external force $f$ generated by its environment. This force will cause the photon to undergo a change in momentum $f=\frac{d p}{d t}=c \frac{d m_{0}}{d t}$. In proceeding against this force it must do work against its environment of amount $f d s=c d m_{0} \frac{d s}{d t}=c^{2} d m_{0}$ The energy it must provide to its 
environment per unit time is $f \frac{d s}{d t}=f_{c}=c^{2} \frac{d m_{0}}{d t}$. Since its energy is $E=\hbar \omega_{0}=m_{0} c^{2}$, its frequency must decrease at the rate $\frac{d \omega_{0}}{d t}=\frac{c^{2}}{\hbar} \frac{d m_{0}}{d t}$.

This may be a slow rate of frequency reduction, but 13.8 billion years is a long time. Cosmic Microwave Background photons, taking part in the Big Bang expansion of the universe for 13.8 billion years can therefore lose a large fraction of their energy and end up with very low frequencies and long wavelengths lying in the microwave region of the energy spectrum. In terms of their blackbody energies, shortly after the explosion, $\mathrm{CMB}$ photons had Planck temperatures of thousands of degrees K. After 13.8 billion years, their blackbody temperature has fallen to $2.74^{\circ} \mathrm{K}$, as observed in the COBE experiment. Throughout 13.8 billion years, $\mathrm{CMB}$ photons have been losing energy without slowing down. The observed phenomenon, experimentally verified by COBE, cannot be explained without Photodynamics. Words alone will not work. Photodynamics provides a physical solution of the CMB photon cooling problem.

Imagine you are cruising the skyways in your automobile. This automobile does not use gasoline or lithium ion batteries. Instead, when it needs energy to escape a planet or meet headwinds, it converts part of its rest mass to energy using $\mathrm{E}=\mathrm{m}_{0} \mathrm{c}^{2}$. You could drive on for billions of years. This is what the photon does.

\section{The Photon's Angular Momentum}

Now for the origin and meaning of $\omega_{0}$. Let $X^{\prime}, Y^{\prime}, Z^{\prime}$ be the coordinates of the photon's center-of- mass in the $R^{\prime}$ lab frame. As we will see, motion in the $x^{\prime}, y^{\prime}$ plane is used to generate the photon's angular momentum, with the result that $\dot{X}^{\prime}=\dot{Y}^{\prime}=0$, and

$$
e_{z^{\prime}}^{2}+c^{2} \bar{p}_{z^{\prime}}^{2}=e_{0}^{2} \text {. }
$$

The internal energy $e_{z^{\prime}}$ then seeks its minimum value $e_{z^{\prime}}^{2}=0$ by generating internal forces per eqn. (14) which accelerate it in the $\pm z^{\prime}$ directions until $p_{z^{\prime}}=m_{0} c$, and the photon is going at the speed of light, $\dot{z}^{\prime}=c$. An example of this kind of motion is given by $\dot{z}^{\prime}=c \tanh \omega_{0} t^{\prime}$. (A dot on a variable in a particular coordinate system refers to the time derivative of that variable using that coordinate system's time variable).

We chose $z^{\prime}$ arbitrarily as the axis of motion, but photodynamics applies equally to all axes, and in fact to all directions. Why does a photon, in free space, go in a straight line in a given direction instead of attempting to go in all directions? In other words, for photon flight, is there a mechanism that plays the role of a rifle barrel in determining its direction of flight, and constraining it to move in that direction?

Fortunately for the photon, there is such a mechanism. It is called angular momentum. So important is angular momentum to photons that they do not exist unless they have angular momentum of $\pm \hbar$ aligned with their direction of flight.

We now move to the photon's rest frame, $\mathrm{R}$, defined as the frame in which its translational velocity is zero. In this frame $\dot{z}$ is zero, but there is residual motion in the plane perpendicular to the $\mathrm{z}$ axis which provides the photon with angular momentum. This motion provides no net motion of the photon's center of mass in the $\mathrm{x}, \mathrm{y}$ plane, so in this frame, the photon's center of mass is at rest. That is $\dot{X}=\dot{Y}=\dot{Z}=0$.

Angular momentum is given by:

$$
\bar{L}=\bar{r} \times \bar{p}
$$

and therefore:

$$
L_{z}=m_{0}(x \dot{y}-y \dot{x}) .
$$

Taking

$$
x=\rho \cos \vartheta, \mathrm{y}=\rho \sin \vartheta,
$$

we get

$$
\begin{aligned}
& \dot{x}=\dot{\rho} \cos \vartheta-\rho \dot{\vartheta} \sin \vartheta \\
& \dot{y}=\dot{\rho} \sin \vartheta+\rho \dot{\vartheta} \cos \vartheta,
\end{aligned}
$$

and therefore

$$
L_{z}=m_{0} \rho^{2} \dot{\vartheta} .
$$

The frequency of a photon, $\omega_{0}$ is a constant which we take to be $\dot{\theta}$. Then since $\dot{L}=\dot{\hbar}=0$, eqn. (20) says $\rho$ must be a constant, which we call $\rho_{0}$. The product $\rho_{0} \omega_{0}$ is a velocity, and the only constant velocity associated with the photon is $\mathrm{c}$, so $\mathrm{c}=\rho_{0} \omega_{0}$. Therefore the magnitude of the photon's angular momentum, from eqn. (20), is:

$$
\hbar=m_{0} \rho_{0}^{2} \omega_{0},
$$

which gives us eqn. (7), $e_{0}=\hbar \omega_{0}=m_{0} c^{2}=c p_{0}$.

From eqn. (19) we get:

$$
\dot{x}^{2}+\dot{y}^{2}=(\rho \dot{\vartheta})^{2}=\left(\rho_{0} \omega_{0}\right)^{2}=c^{2},
$$

so the picture we have is that of a circle of radius $\rho_{0}$ rotating about the flight axis at the speed of light. This is the kinematic picture of the photon. The dynamic picture, the one that produces the physical characteristics of angular momentum $\pm \hbar$ along the flight axis, the energy $\hbar \omega_{0}$, and accelerates the photon to the speed of light, is obtained by populating the circle with the photon's rest mass, $\mathrm{m}_{0}$. That is, we multiply the kinematic picture by $\mathrm{m}_{0}$, as we multiplied the kinematic identity, eqn. (11), by $\mathrm{m}_{0}$ to obtain the photodynamic energy-momentum theorem, eqn. (12). The nature and origin of the photon's rest mass, and of mass in general, is the subject of another paper. Notice that the photon has internal structure and motion that is determined by photodynamics. The photon's internal rotational speed $\dot{x}^{2}+\dot{y}^{2}=c^{2}$ has reduced the internal energy of the energy momentum theorem for the $\mathrm{x}, \mathrm{y}$ plane to zero and produced an energy of motion $\mathrm{cp}=\mathrm{m}_{0} \mathrm{c}^{2}$. This, surprisingly, is the origin of the photon's rest mass, which in the centre-of-mass coordinate system, resides at the centre of the photon, $\mathrm{X}=\mathrm{Y}=\mathrm{Z}=0$. It also constrains motion of the centre of mass in the X,Y plane to $\dot{X}=\dot{Y}=0$, and since XYZ follows the photon, $\dot{Z}=0$ also. It is this rest mass, in the $\mathrm{R}^{\prime}$ lab frame, which accelerates the photon to the speed of light.

Rotation of the photon's mass about the flight axis at the speed of light does not require an object at the center to keep it in orbit. Oscillating motion contributions along the $\mathrm{x}$ and $\mathrm{y}$ axes with speeds ranging between zero and the speed of light generate photodynamic forces whose RMS value and direction are exactly the center directed force required to keep the mass in orbit. Neither is the rotation of a mass element at the speed of light a contradiction of Einstein's $E=m c^{2}$, since $E=m c^{2}$ does not apply to anything that can travel at the speed of light.

Notice that eqns. (21) and (22) hold true in the photon's rest frame, whatever its speed, $\dot{z}^{\prime}$, may be relative to the lab frame. If $\dot{z}^{\prime}$ is not c, the photon's rest energy, or what remains of it, will seek its minimum, in the process accelerating the photon to the speed of light. The rest energy, $e_{z^{\prime}}=m_{0} c^{2}$, can seek its minimum by producing motion along either $\pm z^{\prime}$. That is why the photon has angular momentum $\pm \hbar$ along its flight axis. It is the angular momentum mechanism which defines the axis of flight and serves as the gun barrel for the photon. In a manner 
of speaking, the photon carries its rifled gun barrel with it, and in fact is the gun barrel itself. The mass of the photon circulates endlessly at the speed of light in the rest frame, while the circle itself accelerates down the remaining free rectilinear axis until it reaches the speed of light in the lab frame. It is the rectilinear motion, at the speed of light, which dominates our thinking and not what is going on "inside", and certainly not that this is all made possible by the photon's non-zero rest mass.

\section{Concluding Remarks}

This paper contains a number of findings which we fear might be lost in the presentation. To prevent this, and to address the issues and questions raised in the introduction, we provide a list of the more significant findings. Finally, we provide concluding remarks putting Photodynamics in perspective.

1. Einstein's $\mathrm{E}=\mathrm{mc}^{2}$ has only limited validity. It does not apply to all particles. In particular it does not apply to particles that can travel at the speed of light. This is a breakthrough in relativity.

2. The historical proof, based on $\mathrm{E}=\mathrm{mc}^{2}$, that the photon has a zero rest mass and can only travel at the speed of light is wrong.

3. The fact that the photon's energy $E=\hbar \omega_{0}$ has a non-zero mass equivalence traveling at the speed of light is another proof that $\mathrm{E}=\mathrm{mc}^{2}$ does not apply to photons.

4. The photon has a non-zero rest mass, and can travel at any speed from zero to the speed of light, although it spends most of its time traveling at the speed of light.

5. All particles that travel at the speed of light must have non-zero rest masses in order to exist. This includes photons, neutrinos, gravitons, and gluons. This also means that all particles must have mass in order to exist. This is a breakthrough in particle physics.

6. Two distinct and different energy relations are needed to completely explain particle dynamics. Our photonic energy relation $\mathrm{E}_{1}=\mathrm{m}_{1} \mathrm{c}^{2}=\mathrm{m}_{0} \mathrm{c}^{2} \gamma^{-1}$ provides the dynamics of particles that can travel at the speed of light and forces them to travel at light speed. Einstein's $E_{2}=m_{2} c^{2}=m_{0} c^{2} \gamma$ provides the dynamics of particles that can't travel at the speed of light and prevents them from achieving light speed.

7. The Universe contains two types of particles, those that can and those that can't travel at the speed of light. This cannot be explained without Photodynamics.

8. The existence of two relativistic masses, $m_{1}$ and $m_{2}$, explains why the Universe consists of two types of particles. The belief that the Universe has two types of particles because light speed particles have zero rest masses is wrong.

9. Photodynamics, a new fundamental law of nature, provides the dynamics of particles that travel at the speed of light. This is a breakthrough in physics, in particular, in relativity.

10. For the first time in its history, physics is capable of explaining the motion of all particles in the Universe.

11. The photon is capable of self-propulsion wherein it consumes its internal energy to generate forces which accelerate it in the direction of flight until all its internal energy has been consumed and it has reached the speed of light. This is a breakthrough in particle propulsion and evolution.
12. The photon cannot go faster than the speed of light because it has run out of energy.

13. Photodynamics explains how CMB photons can lose energy for 13.8 billion years and continue to travel at the speed of light. As long as any rest mass remains, any loss of speed is prevented by the self-propulsion mechanism. This is a breakthrough in cosmology.

14. Photodynamics provides the physical mechanism which allows neutrinos to possess non-zero rest masses, travel at the speed of light, and undergo neutrino oscillation at the same time. This is a breakthrough in neutrino astrophysics, one which confronts and resolves the problem posed by the 2015 Nobel Prize in physics.

15. As seen in a different light, the existence of $\mathrm{CMB}$ photon cooling and neutrino oscillation at the speed of light is experimental verification of Photodynamics.

Why did it take so long to make these discoveries? There is nothing wrong with the application of the Einstein equations to most objects and particles encountered in the normal world, since they do not move at the speed of light. If there had been, the error would have been found long ago. The error only occurs when $\mathrm{E}=\mathrm{mc}^{2}$ is applied to light speed particles, most of which are seldom seen. The photon is the only common light speed particle encountered in the normal world, and at light speed behaves the same as predicted in Photodynamics. The other light speed particles are seldom encountered or noticed even though, like the photon, they are instrumental in determining the constituents, structure, and evolution of the Universe.

We expect Photodynamics to impact future research on gravitons, neutrinos, gluons, nuclear physics, general relativity, astrophysics, and cosmology including dark matter and dark energy, as well as fundamental particle physics involving particle interactions, structure, and theories of the origin of particles including string theories.

The Standard Model had to be modified to accommodate massive neutrinos [16]. It will have to be modified again to accommodate massive photons, gluons, and neutrinos traveling at the speed of light. While we are on the subject, we also suggest investigation of the proton radius problem [17] taking into account massive gluons, and of other problems in particle physics taking into account Photodynamics.

In conclusion, we must realize that Photodynamics is the only mechanism that allows particles to travel at the speed of light, and then, only if they have mass. This means that if Photodynamics did not physically exist, then photons, neutrinos, gravitons, and gluons would not exist. QED and QCD would not exist. The fact that they do exist is experimental confirmation that Photodynamics exists and is at work.

Looking at the photon as a prime example of Photodynamics at work, we see that the photon is a truly remarkable, self-contained, self-propelled, self-guided engine ideally suited to carry energy at the speed of light. It is difficult to imagine a better mechanism than Photodynamics to get this done. The energy the photon carries is used, in a non-dissipative fashion, to maintain light speed. Because of its simple, non-dissipative design, it is not surprising that it is an abundant, ubiquitous particle that plays a central role in the creation of the universe, the transportation of energy from stars to planets, and the emergence of all types of life. We must not forget that it is the force carrier for electromagnetism. Without the photon there would be no electromagnetic force, and therefore no electrons, protons, 
Citation: Martineau RJ (2018) Photodynamics: How Massive Photons, Gravitons, Gluons, and Neutrinos Manage to Travel at the Speed of Light. J Phys Math 9: 293. doi: 10.4172/2090-0902.1000293

Page 8 of 8

atoms, molecules, human beings, planets, or stars. The galaxies would not exist, and the universe, as a collection of galaxies and the space in between would not exist. So we owe our existence, all that we see, and have come to know to the existence of the photon, which would not exist without its rest mass. All the above would be impossible if the photon did not have Photodynamics to fall back on where Einstein's $\mathrm{E}=\mathrm{mc}^{2}$ fails. Photodynamics has been working in the background, unknown, to make all this possible.

\section{References}

1. Green B (2011) The Hidden Reality. (New York: Alfred A Knopf).

2. Alpher RA, Herman RC (1948) Evolution of the Universe. Nature 162: 774-775.

3. Mather JC, Boslough J (2008) The Very First Light. (New York: Basic Books).

4. Cleveland BT, Daily T, Davis RJr, Distel JR, Lande K, et al. (1998) Measurement of the Solar Electron Neutrino Flux with the Homestake Chlorine Detector. Astrophysics Journal 496: 505-526.

5. Bahcall JN (1964) Solar Neutrinos I. Theoretical. Phys Rev Lett 12: 300.

6. Davis RJr (1964) Solar Neutrinos II. Experimental. Phys Rev Lett 12: 303.

7. Davis RJr, Harmer DS, Hoffman KC (1968) Search for Neutrinos from the Sun Phys Rev Lett 20: 1205.
8. Pontecorvo BM (1967) Neutrino Experiments and the Problem of Conservation of Leptonic Charge. Th Exsp Theor Fiz 53: 1717-1725.

9. Gribov VN, Pontecorvo BM (1969) Neutrino Astronomy and Leptonic Charge. Phys Rev Lett B 28: 493-496.

10. Wolfenstein L (1978) Neutrino Oscillation of Atmospheric Neutrinos in Matter. Phys Rev D 17: 2369.

11. Fukada Y (1998) Evidence for Oscillation of Atmospheric Neutrinos. Phys Rev Lett 81: 1562.

12. McDonald AB, Klein JR, Wark DL (2003) Solving the Solar Neutrino Problem. Scientific American 288.

13. Ahmad QR, et al. (2001) Measurement of the Rate of $v+d \rightarrow p+p+e-$ Interactions Produced by ${ }^{8} \mathrm{~B}$ Solar Neutrinos at the Sudbury Neutrino Observatory. Phys Rev Lett 87: 071301.

14. Kalbfleisch B, Baggett N, Fowler EC, Alspector J (1979) Experimental Comparison of Neutrino, Antineutrino, and Muon Velocities. Phys Rev Lett 43: 1361.

15. Longo MJ (1987) Tests of Relativity from SN1987A. Phys Rev D 36: 3276

16. Yang CN (2003) Neutrinos and Implications for Physics Beyond the Standard Model. (Singapore: World Scientific).

17. Bernauer JC, Pohl R (2014) The Proton Radius Problem. Scientific American 310: 32 . 